\title{
The Effects of Heat-Release during the Nucleation of Dibutylphthalate Nanoparticles in a Laminar Jet
}

\author{
Sean Garrick \\ Department of Mechanical Engineering, University of Minnesota \\ 111 Church Street SE, Minneapolis, USA \\ sgarrick@umn.edu
}

\begin{abstract}
The effect of neglecting heat release during the modeling and simulation of nanoparticle nucleation from vapor are studied. It is common to neglect heat release when modeling particle formation and growth. This allows the use of a single transport equation to represent both the energy and the vapor concentration, and typically results in a significant decrease in compute-time. Using dibutylpthalate (DBP) as a model material, direct numerical simulations of nanoparticle nucleation are performed. The flows consist of hot DBP vapor, diluted in nitrogen $\left(N_{2}\right)$ issuing into a cooler $N_{2}$ environment. As the vapor cools, molecules collide and grow, forming stable clusters which leads to particle nucleation. Simulations are performed with and without accounting for the heat-release during the exothermic process. The results show that the effect of heat release is significant as it decreases particle nucleation rates by up to three orders of magnitude and it increases the size of the particles formed by roughly thirty percent.
\end{abstract}

Keywords: Nanoparticle, nucleation, heat-release, modeling, simulation

\section{Introduction}

Modeling the formation of nanoparticles from vapor is a challenging problem for scientists and engineers interested in gas-to-particle conversion processes. The first step in homogeneous systems, is the nucleation. Nucleation may be thought of as the interplay of temperature and species/vapor concentration that produces a stable cluster [1]. A byproduct of the particle formation is the liberation of energy via heat. From a fluid dynamics perspective, nucleation is very sensitive to the transport of energy and mass or temperature and concentration. A not uncommon modeling strategy is to utilize a single transport equation to represent both temperature and species mass [3, 9]. Such an approach simplifies modeling and reduces the compute time in performing the simulations. One side effect of this formulation is that the energy released during nucleation cannot be taken into account and the effect of this energy transfer on the nucleation process is not clear. Previous research has shown that energy release during nanoparticle during collision and coalescence can have significant effects on subsequent processes [5].

We simulate the nucleation of dibutylpthalate (DBP) particles via direct numerical simulation in a planar jet. We perform the simulations with and without the heat-release that accompanies the nucleation process. Our objective is to examine how the heat-release affects the rate of particle nucleation as well as the size of the particles formed.

\section{Formulation}

The flows under consideration are governed by the compressible Navier-Stokes equations, the primary variables of which are $u_{i}$, the velocity in the $x_{i}$ direction, the pressure, $p$, the fluid density, $\rho$, and the enthalpy, $h$. The transport equations for the mass, momentum and enthalpy are omitted for brevity but they are available elsewhere [6]. The equations are closed using the ideal gas relation, $p=\rho R T$, where $R$ is the gas constant, and $T$ is the temperature. The enthalpy, $h$, is related to $T$ via $d h=C_{p} d T$, where $C_{p}$ is the specific heat. The flows consist of DBP vapor diluted in nitrogen $\left(N_{2}\right)$. Each species is represented by its mass fraction, $Y_{i}$, and the transport equation is given by

$$
\frac{\partial \rho Y_{i}}{\partial t}+\frac{\partial \rho u_{j} Y_{i}}{\partial x_{j}}=\frac{\partial}{\partial x_{j}}\left(\rho D_{i} \frac{\partial Y_{i}}{\partial x_{j}}\right)+\dot{\omega}_{i}
$$


where $D_{i}$ is the diffusion coefficient of species $i$, the term $\omega_{i}$ represents vapor-to-particle mass conversion (via nucleation) and is given by

$$
\dot{\omega}_{i}=\frac{\pi}{6} \rho_{p} d_{c}^{3} J
$$

where $\rho_{p}$ is the density of the DBP particle, $d_{c}$ is the critical particle diameter, and $J$ is the DBP nucleation rate. The enthalpy increase by nucleation per unit time is given by $Q=\omega_{i} h_{o}$, where $h_{o}$ is the enthalpy of formation of DBP particle.

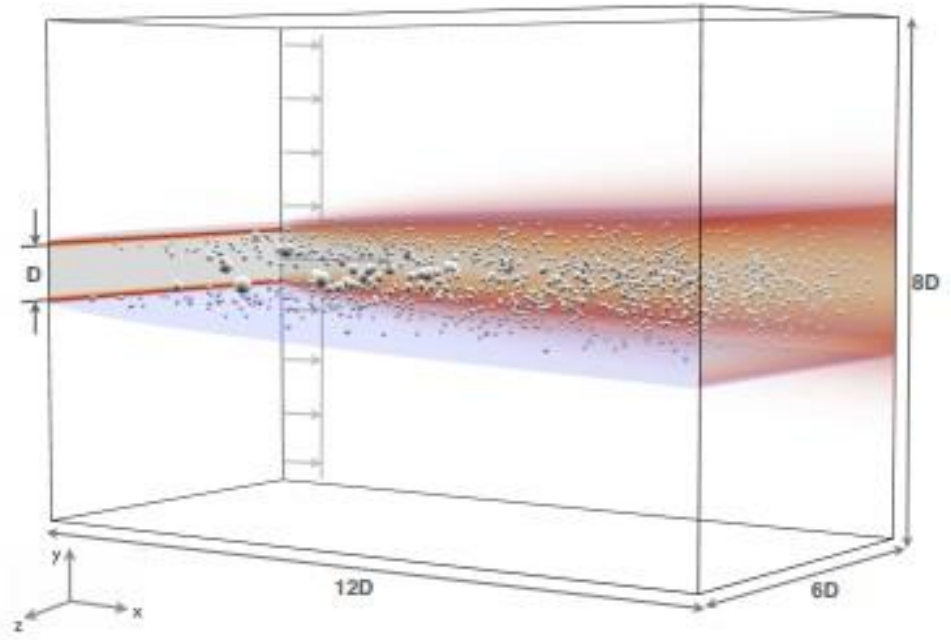

Fig. 1: Flow configuration with vorticity magnitude contour at plane $y=0$ and $z=0$. The size of the spheres are scaled to the critical diameter and the number density is scaled to the DBP nucleation rate in case 1.

The nucleation of DBP is modeled via classical nucleation theory (CNT) [1]. Using CNT, the nucleation rate is given by

$$
J=\frac{\left(\rho Y_{D B P}\right)^{2}}{\rho_{p} m_{p}} \sqrt{\frac{2 \sigma}{\pi m_{p}}} \exp \left(-\frac{\pi \sigma d_{c}^{2}}{3 k_{B} T}\right),
$$

where $Y_{D B P}$ is the DBP mass fraction, $m_{p}$ is the mass of a DBP molecule, $\sigma$ is the surface tension, and $k_{B}$ is the Boltzmann constant. Similarly the size of the critical nuclei, $d_{c}$, is given by the Kelvin relation

$$
d_{c}=\frac{4 \sigma m_{p}}{k_{B} T \rho_{i} \ln (S)} .
$$

We define a nucleation Damkohler number, $D a_{N}$, to characterize the ratio of the nucleation to convective time scales [4]. It is a function of the flow and material properties at the reference conditions and given by

$$
D a_{N}=\frac{L_{o}}{6 U_{o} \rho_{o}}\left(\frac{\left(\rho_{o} Y_{o}\right)^{2}}{m_{p}^{3 / 2}} \sqrt{2 \pi \sigma_{o}}\right)\left(\frac{\sigma_{o} m_{p}}{\rho_{p} k_{B} T_{o}}\right)^{3} .
$$

This definition is useful in closing the problem using non-dimensional parameters and in characterizing different regimes in turbulent flows involving nucleation ([2]). 


\section{Results}

The flows under consideration consist of a three dimensional planar jet with velocity $U_{o}$, laden with DBP vapor at temperature $T_{o}=400 \mathrm{~K}$ issuing through a slot of height $D$ into co-flowing nitrogen stream with velocity $U_{\infty}$ at temperature $T_{\infty}=300 \mathrm{~K}$. The Reynolds number is $\operatorname{Re}=U_{o} D / v=200$, the Prandtl number is $\operatorname{Pr}=1$ and the Lewis number is $\operatorname{Le}=\operatorname{Sc} / \operatorname{Pr}=$ 4. All physical properties are calculated based on the local temperature, $T$, and the mass fraction of each chemical species (DBP and $N_{2}$ ). DBP properties are taken from previous research $([7,8])$. The DBP condensed phase density is given by $\rho_{p}$ $=1063-0.826(T-273.16) \mathrm{kg} / \mathrm{m}^{3}$, its surface tension is $\sigma=0.0353-0.0000863(T-273.16) \mathrm{N} / \mathrm{m}$. The saturation ratio is given by

$$
S=\frac{M W_{\text {mix }}}{M W_{D B P}} \frac{Y_{D B P}}{x_{\text {sat }}}
$$

and the saturation mole fraction is given by $x_{\text {sat }}=\exp (21.497-11497 / \mathrm{T})$. We perform three simulations with different nucleation Damkohler numbers: case $1-D a_{N}=206.8 \times 102$; case $2-D a_{N}=206.8 \times 106$; and case $3-D a_{N}=206.8 \times 109$.

The governing equations are solved using a second order in time, fourth order in space, predictor-corrector scheme. Computations are performed on a domain of $12 D \times 8 D \times 6 D$ in the $\mathrm{x}, \mathrm{y}$ and $\mathrm{z}$ directions respectively. The resolution for all simulations is $320 \times 240 \times 120$. The grid is clustered about the shear layers and each simulation performed requires roughly 300 CPU-hours.
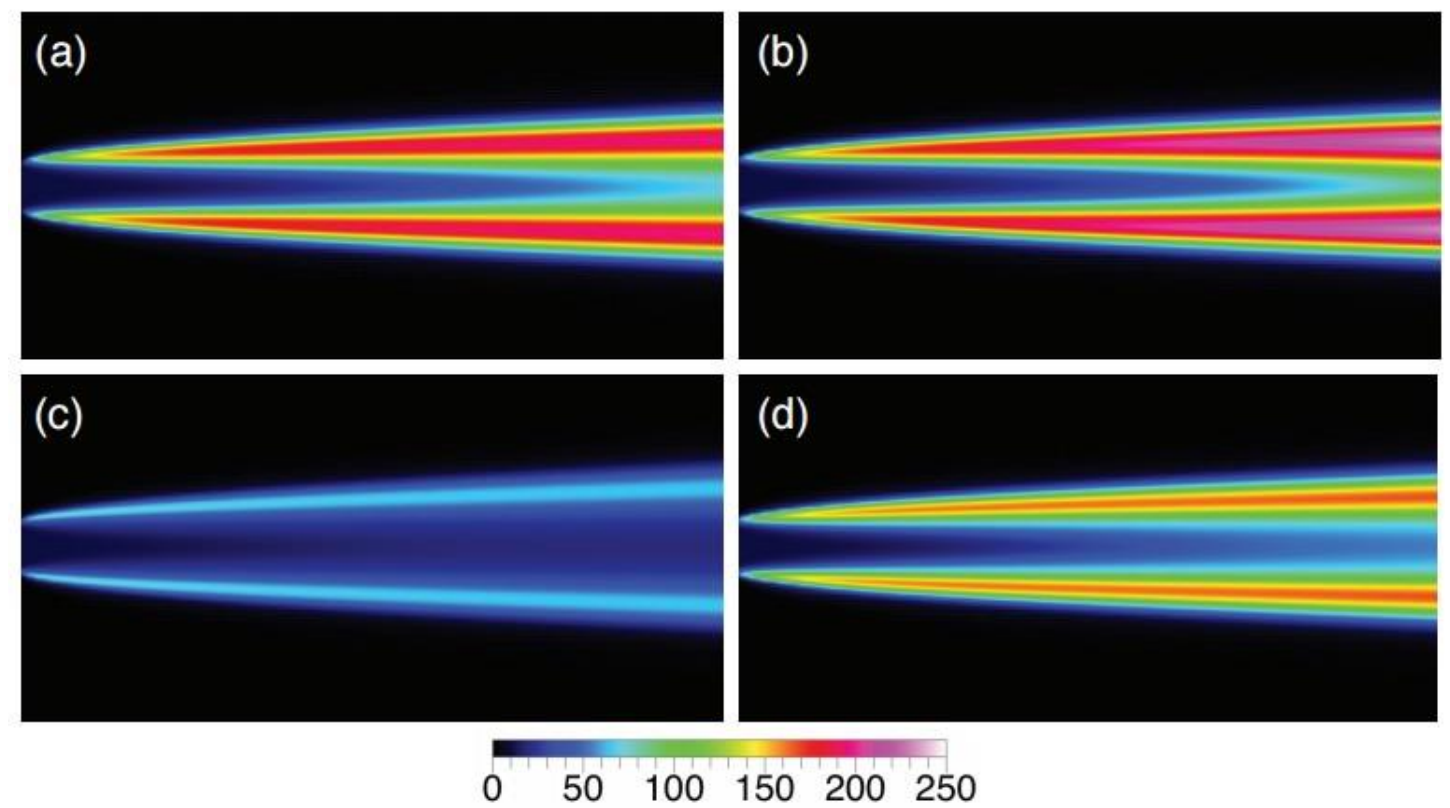

Fig. 2: Contours of the saturation ratio, S: (a) case 1, w/ heat-release; (b) case 1, no heat-release; (c) case 3, w/ heat-release; (d) case 3 , no heat-release.

The saturation ratio $S$, acts as a bridge between scalar transport and particle nucleation. It is the ratio of the condensing vapor partial pressure to its saturation pressure. Contours of the saturation ratio $S$ are shown in Fig. 2. The results for case 1 $\left(D a_{N}=206.8 \times 10^{2}\right)$ with and without nucleation heat-release, are shown in Fig. 2(a) and Fig. 2(b), respectively. The results for case $3\left(D a_{N}=206.8 \times 10^{9}\right)$ with and without heat-release effects are shown in Fig. 2(c) and Fig. 2(d), respectively. The effect of heat-release on the saturation ratio in case is relatively mild. The heat-release has the effect of increasing the temperature and decreasing the saturation ratio. When taking heat-release into account in case 1 , the peak value of the saturation ratio is $10 \%$ less $(S=200$ compared to $S=220)$. In case 3 , the disparity is much greater. With heatrelease, the peak saturation ratio is roughly $S=65$ and without heat-release the saturation ratio is roughly $S=160$. I.e. heatrelease causes the saturation ratio to drop by $60 \%$ in case 3 . In CNT, the saturation ratio is important only inasmuch as it affects the nucleation rate and critical cluster size. 
Scatter plots of the nucleation rate, $J$, vs. critical diameter, $d_{c}$, for all 3 cases are shown in Fig. 3 . The results with heat release are in red and the results without heat release is in black. In all cases, the smallest particles are formed at the highest rate as previously observed ([6]). As the nucleation Damkohler number increases, the rate of particle nucleation increases and the critical diameter decreases. However, the results show that with heat-release taken into account, the degree to which the nucleation rate increases with the Damkohler number is lessened. For example, with heat-release, the peak nucleation rate is roughly $J=10^{21}$ particles $/\left(\mathrm{m}^{3} \cdot \mathrm{s}\right)$ in case 1 and $J=10^{22}$ particles $/\left(\mathrm{m}^{3} \cdot \mathrm{s}\right)$ in cases 2 and 3. However, without heat-release the peak nucleation rates are $J=10^{22}$ particles $/\left(\mathrm{m}^{3} \cdot \mathrm{s}\right), J=10^{26}$ particles $/\left(\mathrm{m}^{3} \cdot \mathrm{s}\right)$, and $J=10^{27}$ particles $/\left(\mathrm{m}^{3} \cdot \mathrm{s}\right)$ in cases 1,2 and 3 , respectively.

A similar trend is observed in the critical cluster size, $d_{c}$. When heat-release is not taken into account, the critical size decreases. Larger critical nuclei are formed with heat-release because the increased kinetic energy of molecules needs to be balanced by larger surface to be stable for future growth. Also, when heat release is not taken into account the critical cluster size increases with nucleation Damkohler number. This is because higher Damkohler numbers decreasing DBP mass-fraction - due to vapor-to-particle - and increasing temperature as energy is release during nucleation. As a result, when heat-release is not taken into account the rate of particle nucleation is over-predicted.

\section{Conclusions}

We performed simulations of DBP nanoparticle nucleation in laminar jets. We solved the Navier-Stokes equations along with the conservation of enthalpy and DBP mass-fraction transport equation. We examined the effects of heatrelease by performing simulations with and without the change in enthalpy that accompanies gas-to-particle nucleation at different nucleation Damkohler numbers.
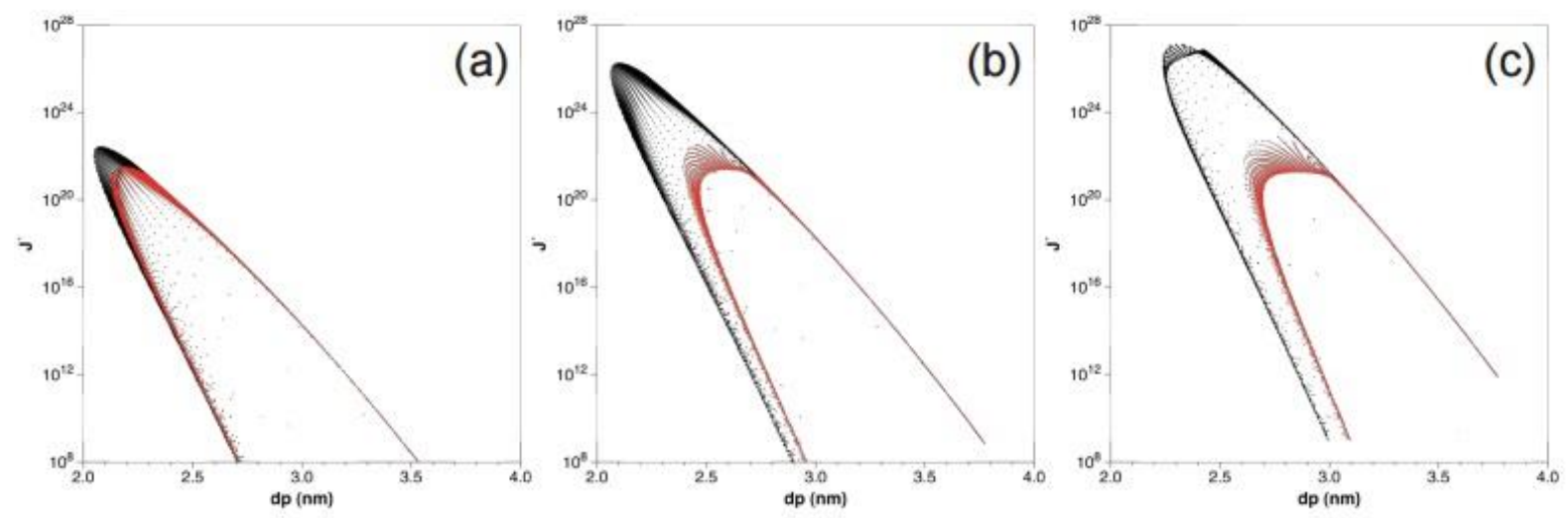

Fig. 3: Nucleation rate $J$ vs. critical diameter $d_{p}$. The results in red (light) correspond the simulations with heat-release and those in black (dark) correspond to simulations without heat-release: (a) $D a_{N}=206.8 \times 10^{2}$; (b) $D a_{N}=206.8 \times 10^{6}$; (c) $D a_{N}=206.8 \times 10^{9}$.

The results show that at low Damkohler numbers the effect of heat-release on the saturation ratio is slight, indicating the amount of heat-release during nucleation is negligible. The nucleation rate differs by 1 order of magnitude and the critical diameter varies by 5\%. However, as the Damkohler number increases, the saturation ratio is greatly over-predicted when heat-release is neglected. It has a similar effect on the nucleation rate. Neglecting heat-release during nucleation results in an artificially increased rate of particle formation - by as much as 4 orders of magnitude in our study - and the diameter of the nucleating particles is under-predicted by as much as $21 \%$.

The numerical schemes used are second order accurate in time and fourth order accurate in space. A grid refinement study was performed until the predictions/results varied by less than $0.01 \%$. As a result the numerical predictions are reliable. However, the trends observed should be taken in several contexts. First, for the results to hold, classical nucleation theory must provide an accurate representation of the nucleation process. Second, the disparities will decrease as the mass fraction of the nucleating material decreases. These results are particularly pertinent in modelling synthesis reactors, where performance characterization is important. Results show that accurate prediction requires the solution of separate energy and mass-fraction (or concentration) equations. 


\section{Acknowledgments}

All computations were performed at the Minnesota Supercomputing Institute.

\section{References}

[1] K. S. Friedlander, Smoke, Dust and Haze: Fundamentals of Aerosol Dynamics. Oxford University Press, New York, NY, 2000.

[2] C. Helling, R. Klein, M. Luttke, and E. Sedlmayr, (2000). Dust formation in turbulent media. In H. Freistjhler, and G. Warnecke, editors, Hyperbolic Problems: Theory, Numerics, Applications: Proceedings of the Eighth International Conference in Magdeburg, number 141 in International Series of Numerical Mathematics, pp. 515-524, Basel, CH. Birkhauser.

[3] C. Housiadas, Y. Drossinos, and M. Lazaridis, "Effect of small-scale turbulent fluctuations on rates of particle formation," J. Aerosol Sci., vol. 35, pp. 545-559, 2004.

[4] V. V. Kelkar, and K. M. Ng, "Design of reactive crystallization systems incorporating kinetics and mass-transfer effects," AIChE J., vol. 45, pp. 69-81, 1999.

[5] K. E. J. Lehtinen, and M. R. Zachariah, "Energy accumulation in nanoparticle collision and coalescence processes," J. Aerosol Sci., vol. 33, pp. 357-368, 2002.

[6] N. J. Murfield, J. Pyykonen, J. Jokiniemi, and S. C. Garrick, "The structure of nanoparticle nucleation in planar jets," J. Aerosol Sci., vol. 62, pp. 1-14, 2013.

[7] H. V. Nguyen, K. Okuyama, T. Mimura, Y. Kousaka, R. Flagan, and S. Seinfeld, "Homogeneous and heterogeneous nucleation in a laminar flow aerosol generator," J. Colloid Interf. Sci., vol. 119, pp 491-504, 1987.

[8] J. Pyykonen, and J. Jokiniemi, "Computational fluid dynamics based sectional aerosol modelling schemes," J. Aerosol Sci., vol. 31, pp. 531-550, 2000.

[9] K. Zhou, and T.-L. Chan, "Simulation of homogeneous particle nucleation in a free turbulent jet," Aerosol Sci. and Technol., vol. 45, pp. 973-987, 2011. 